\title{
Uma Abordagem Integrada de Hardware e Software para o Ensino de Organização e Arquitetura de Computadores
}

\author{
Cícero Samuel Mendes ${ }^{1}$, Lucas Fontes ${ }^{1}$, Edson Lisboa ${ }^{2}$, Guilherme Esmeraldo ${ }^{1}$ \\ ${ }^{1}$ Instituto Federal de Educação, Ciência e Tecnologia do Ceará - Crato \\ ${ }^{2}$ Instituto Federal de Educação, Ciência e Tecnologia de Sergipe - Aracaju \\ mendes.samuel99@gmail.com, lfonteesc@gmail.com, \\ edson.lisboa@academico.ifs.edu.br, guilhermealvarodifce.edu.br
}

\begin{abstract}
This paper presents a simulator-based approach which is integrated to an open prototyping platform to support the teaching-learning processes and development of practical skills in the discipline of Computer Organization and Architecture. The results show the effectiveness of the learning process and the increase of students motivation and performance by the use of the proposed simulator.
\end{abstract}

Resumo. Este artigo apresenta uma nova abordagem baseada em simulador integrado a plataforma aberta de prototipação para apoio aos processos de ensino-aprendizagem e desenvolvimento de habilidades práticas na disciplina de Organização e Arquitetura de Computadores. Os resultados mostram a efetividade no aprendizado e aumenta de motivação e desempenho dos estudantes pelo uso do simulador proposto.

\section{Introdução}

O avanço tecnológico e a alta escala de integração têm contribuído para o desenvolvimento de plataformas computacionais cada vez mais compactas com a finalidade de atender às mais variadas necessidades de mercado e pesquisa, abrangendo desde soluções para computação de alto desempenho a sistemas embarcados. Por outro lado, essas características agregam ainda mais complexidade à disciplina de Organização e Arquitetura de Computadores (OAC), tornando-a uma das mais desafiadoras em cursos de Ciência e Engenharia da Computação, uma vez que precisa abordar, de forma teórica e prática, diferentes aspectos de hardware, software de baixo nível e interfaceamento cada vez mais elaborado.

Este trabalho apresenta uma nova abordagem para ensino de OAC, a qual faz uso de um simulador computacional integrado a uma plataforma aberta de prototipação para apoiar o aprendizado dos conteúdos e o desenvolvimento das habilidades práticas da disciplina. 
VII Congresso Brasileiro de Informática na Educação (CBIE 2018)

Anais do XXIX Simpósio Brasileiro de Informática na Educação (SBIE 2018)

\section{Trabalhos Relacionados}

Novas abordagens metodológicas têm sido propostas, na literatura, para dar suporte ao processo de transferência de conhecimento em OAC. O trabalho em [Fernandes; Silva, 2017] cita diferentes abordagens para o ensino de OAC, como são os casos de Problem Based Learning, Ensino baseado em Projetos, em Analogia, em Aplicação, entre outras. Da análise delas, pode-se concluir que o emprego de situações-problema e de atividades práticas aplicadas favorecem o aprendizado em OAC, porém necessitam de ferramental adequado. Nesse sentido, tem-se adotado o uso de simuladores computacionais como ferramenta de apoio pedagógico [Fernandes; Silva, 2017]. Vários estudos, como o apresentado em [Penna; Freitas, 2013], realizaram comparativos entre simuladores da literatura, onde consideraram determinadas métricas comparativas, como, por exemplo, alto desempenho, suporte de interface gráfica, disponibilidade de documentação, distribuição livre, entre outras. Observa-se todavia que os simuladores: ou não apresentam todos os recursos didáticos necessários para apoio à disciplina [Penna; Freitas, 2013]; ou, devido à adoção de abstrações, não abordam adequadamente conteúdos importantes [Larraza-Mendiluze; Garay-Vitoria, 2015]; ou, por focarem em determinados componentes do computador, há a necessidade de se utilizar mais de um simulador [Fernandes; Silva, 2017]; ou buscam fidelizar as características dos componentes do computador, tornando-se complexos de configurar e interagir [Duenha; Azevedo, 2016]. Além de um simulador versátil e de fácil uso, é fundamental que, em algum ponto da abordagem metodológica, o estudante tenha contato com hardware real, pois, além de aumentar a motivação para estudos avançados em OAC, terá que lidar com eles durante o exercício da profissão [Black, 2016].

Neste trabalho, o simulador utilizado, chamado de CompSim, está integrado à plataforma aberta de prototipação Arduino, os quais são utilizados conjuntamente em práticas laboratoriais da disciplina para apoio ao aprendizado em OAC e oferecer aos estudantes diferentes cenários de contato com hardware.

\section{A Abordagem de Ensino com Apoio de CompSim e Arduino}

Considerando as diferentes ementas, contextos locais e cursos de computação, a disciplina de OAC pode sofrer variações quanto à extensão e verticalização de seus conteúdos. Desta forma, na abordagem aqui apresentada, o uso do simulador CompSim trata de um subconjunto de conteúdos da disciplina comuns a esses cursos, tais como: introdução à aritmética computacional, componentes do computador e suas funções, conjunto de instruções do processador, modos de endereçamento, entrada/saída, programação em baixo nível e análise de desempenho.

O simulador CompSim possui uma interface gráfica que permite configurar diferentes componentes virtuais de hardware, suportar o desenvolvimento de aplicações e realizar simulações. Entre os componentes que oferece, estão: processador, memórias RAM e cache, barramentos e periféricos de vídeo e teclado. A Figura 1 mostra a 
VII Congresso Brasileiro de Informática na Educação (CBIE 2018)

Anais do XXIX Simpósio Brasileiro de Informática na Educação (SBIE 2018)

interface gráfica do CompSim, onde pode-se visualizar: A) editor de código; B) processador; C) memória cache; D) memória RAM; E) vídeo; F) teclado e G) componentes de configuração e execução de simulação.

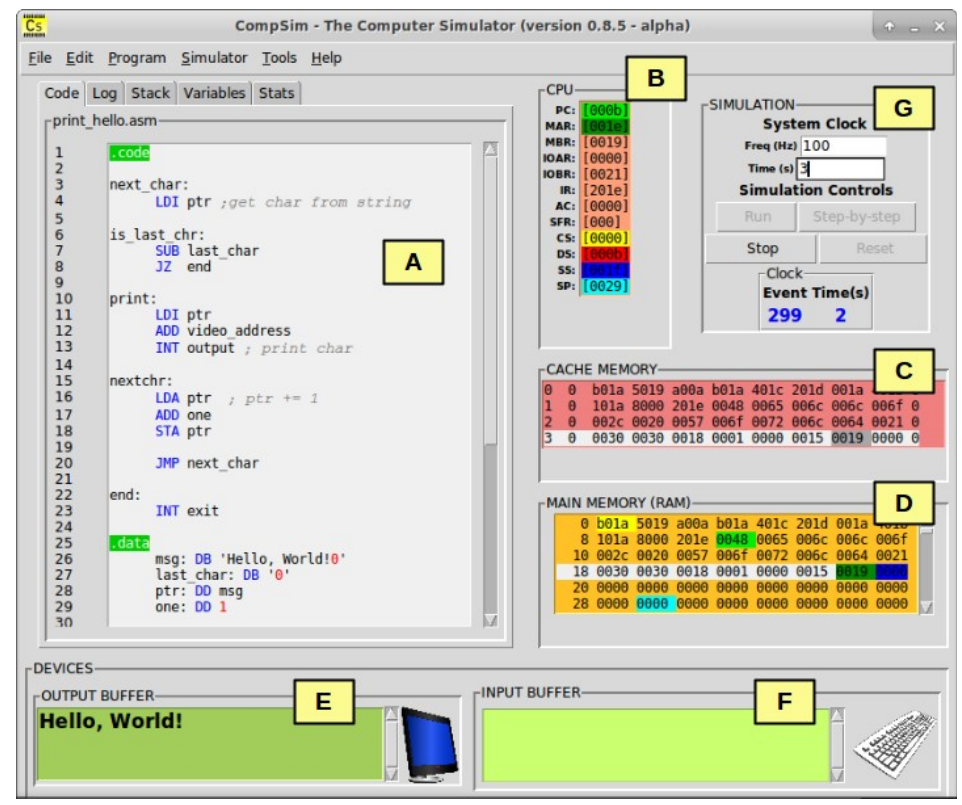

Figura 1. Interface gráfica do simulador CompSim.

Para estimular o contato com o hardware real, a integração com a plataforma Arduino permite que os estudantes possam desenvolver dispositivos eletrônicos, de forma simplificada [Egea; Rosa; Rodriguez, 2017], e integrá-los aos componentes virtuais do CompSim, compondo assim sistemas computacionais complexos. Desta forma, durante o desenvolvimento da disciplina, podem ser oferecidos diferentes cenários para o desenvolvimento de dispositivos, onde os estudantes podem manusear componentes eletrônicos como resistores, capacitores, sensores, motores, leds, displays, chaves táteis, entre outros, para a criação de novos dispositivos integrados ao CompSim. Entre os cenários propostos estão: "pisca leds" (“árvore-de-natal”, semáforos e jogos), exibição de dados em displays (contadores numéricos, relógio e menu interativo), monitoramento (temperatura, umidade, luminosidade, proximidade e infravermelho), sinalizadores (alarme e código morse) e movimento (hélices, carrinho e braço mecânico).

A Figura 2 ilustra, através de um fluxograma, as etapas da abordagem de ensino proposta neste artigo. As etapas em cor branca estão relacionadas às aulas onde há exposição puramente teórica dos conteúdos da disciplina de OAC; as etapas em cor cinza estão relacionadas às aulas práticas em laboratório com suporte do simulador CompSim; e as etapas em cor amarelo abrangem às aulas práticas em laboratório com suporte do simulador CompSim e de plataformas Arduino. Ressalta-se que, neste último grupo de atividades, deverão estar disponíveis para os estudantes, além do simulador, kits compostos por plataformas Arduino (UNO ou MEGA) e por componentes 
VII Congresso Brasileiro de Informática na Educação (CBIE 2018)

Anais do XXIX Simpósio Brasileiro de Informática na Educação (SBIE 2018)

eletrônicos, necessários à realização dos cenários propostos pelo professor da disciplina.

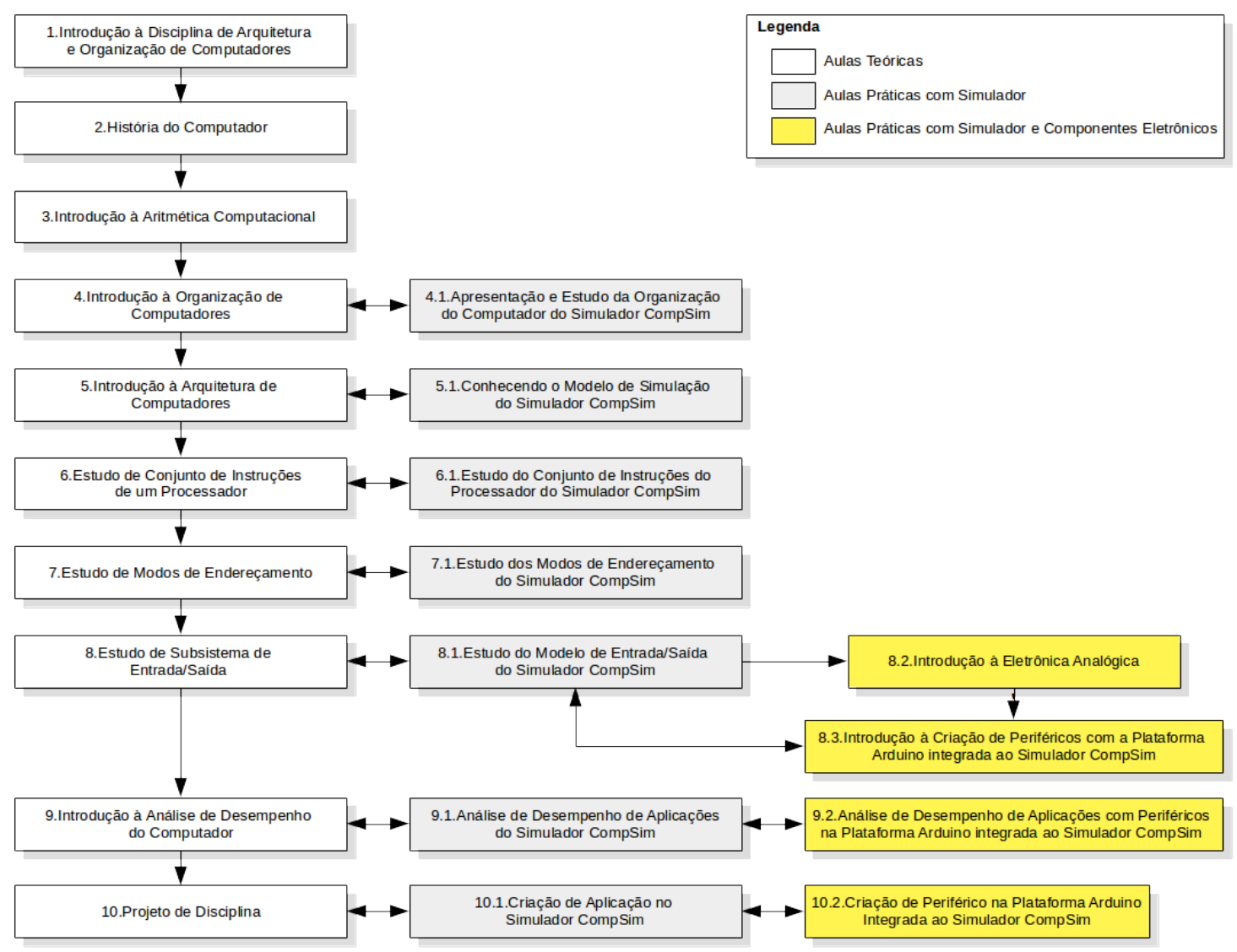

Figura 2. Fluxograma da abordagem de ensino proposta.

\section{Resultados Experimentais}

O CompSim tem sido utilizado e avaliado por turmas de OAC do curso de bacharelado em Sistemas de Informação do Instituto Federal de Educação do Ceará campus Crato. Ao final de cada semestre letivo, os estudantes preenchem uma rubrica, onde são avaliados o "Suporte Educacional" e a "Experiência de Uso" do simulador CompSim. Nos resultados das avaliações, até o presente momento, os estudantes qualificaram, em média, como "Excelente" e "Bom" o suporte educacional e a experiência de uso, respectivamente. Cabe ressaltar ainda que os estudantes têm destacado positivamente o suporte ao pensamento de alto nível e a efetividade no ensino pelo uso do simulador. Além disso, foi possível constatar um aumento significativo na motivação e desempenho geral da turma, de através da comparação entre médias de frequência/participação dos estudantes em aulas práticas em laboratório e de conceitos acadêmicos atribuídos às turmas, respectivamente, em relação às turmas que não tiverem contato com o simulador.

Atualmente, a abordagem proposta neste artigo está sendo aplicada a uma nova 
VII Congresso Brasileiro de Informática na Educação (CBIE 2018)

Anais do XXIX Simpósio Brasileiro de Informática na Educação (SBIE 2018)

turma da disciplina de $\mathrm{OAC}$, onde ainda não foi possível avaliar a efetividade do aprendizado baseado em cenários de uso de hardware real através da integração CompSim-Arduino.

\section{Conclusões}

Abordagens baseadas em uso de simuladores têm se mostrado bastante efetivas nos processos de ensino-aprendizagem de OAC. No entanto, é importante que os estudantes tenham contato com hardware real para desenvolverem as habilidades práticas necessárias à profissão. Nesse cerne, o uso de simulador integrado a uma plataforma aberta de prototipação constitui-se uma opção viável, desde que podem ser projetados desde simples dispositivos eletrônicos até sistemas computacionais complexos. Como trabalhos futuros, espera-se validar a abordagem aqui proposta, além de estendê-la para oferecer um comportamento multidisciplinar.

\section{Referências}

Black, M. (2016) "Export to arduino: a tool to teach processor design on real hardware". Journal of Computing Sciences in Colleges, 31(6). pp. 21-26.

Duenha, L.; Azevedo, R. (2016) "Utilização dos Simuladores do MPSoCBench para o Ensino e Aprendizagem de Arquitetura de Computadores". In: International Journal of Computer Architecture Education (IJCAE), V. 5, n. 1. pp 26-31.

Egea, R; Rosa., R.; Rodriguez, D. Z. (2017) “An Open Electronic Prototyping Platform as Resource for Teaching”. In: Simpósio Brasileiro de Informática na EducaçãoSBIE. p. 1739.

Fernandes, S. R.; Silva, I. S. (2017) "Relato de Experiência Interdisciplinar Usando MIPS”. In: International Journal of Computer Architecture Education (IJCAE), V. 6, n. 1. pp. 52-61.

Larraza-Mendiluze, E.; Garay-Vitoria, N. (2015) "Approaches and tools used to teach the computer input/output subsystem: A survey". IEEE Transactions on Education, v. 58 , n. 1, p. $1-6$.

Penna, P. H; Freitas, H. C. (2013) "Análise e Avaliação de Simuladores de Sistemas Completos para o Ensino de Arquitetura de Computadores". In: Int. Journal of Computer Architecture Education, V. 2, N.1. pp. 13-16. 